\title{
Autoetnografías de descolonización: de cómo dos investigadores latinoamericanos nos comenzamos a descolonizar en el Norte global
}

\author{
VICTORIA MARÍN-BURGOS* \\ ÓSCAR R. ENRÍQUEZ PAZ Y PUENTE**
}

\section{INTRODUCCIÓN: CECI N'EST PAS UN «PAPER» (MAGRITTE 1928-1929)}

Esto no es un paper, es un ensayo, un diálogo aparentemente improbable entre dos investigadores recientemente doctorados en una universidad holandesa, una en estudios del desarrollo y otro en física de fluidos (minúsculas intencionales). Se trata de un intento por tomar distancia de nuestros quehaceres científicos, observarlos y reflexionar al respecto con sensibilidad autoetnográfica.

El asunto del que nos ocupamos en este ensayo tiene que ver con la práctica de una ciencia para el Sur y desde el Sur (nos referimos aquí a los países del llamado Sur global). Basándonos en nuestra experiencia como investigadores en una universidad del Norte global, cuestionamos la hegemonía del modelo de ciencia occidental en relación con las realidades y el quehacer científico en nuestra América Latina, y presentamos algunas reflexiones sobre la descolonización de la ciencia.

Siendo un ensayo, permítasenos comenzar citando un texto que expresa bien la naturaleza de este género:

*Doctora en Estudios del Desarrollo y Ecología Política por la Universidad de Twente, Países Bajos.

**Doctor en Mecánica de Fluidos por la Universidad de Twente, Países Bajos. 
El ensayo duda de sí mismo incesantemente. Como género surgió preñado de escepticismo, y su significado etimológico evoca ya el ser un intento, jamás una certeza. Es un ejercicio de preguntas que se dejan al aire, no para responderse ahí mismo, quizás en otro instante, quizás 10 ensayos más adelante. El ensayo es ambivalente, afirma y duda en el mismo espacio; el ensayo es, ante todo, un ejercicio lúdico. Es intento, no definición, no verdad; es un baile, un juego de las ideas, una aproximación lúdica a la producción del conocimiento (Azahua, 2013: 15).

Así pues, asumiendo el carácter de un ensayo, este texto no pretende demostrar la veracidad o falsedad de una hipótesis, ni responder a una pregunta de investigación. Más bien se presenta como un ejercicio de reflexión y diálogo a través del cual nos planteamos muchas preguntas que quedan abiertas a debate y esbozamos algunas ideas sujetas a discusión.

Para poner en práctica eso de la sensibilidad autoetnográfica, recurrimos a algo que sí es un instrumento de la investigación académica en el campo de las ciencias sociales: la autoetnografía. Es decir, hemos asumido el papel de un investigador,

[...] que se enfoca intensamente en sus propias circunstancias de vida como una forma de entender fenómenos sociales o culturales mayores y que frecuentemente recurre en sus textos a un estilo de narrativa personal como estrategia figurativa que incorpora afectos y emociones al análisis (Butz y Besio, 2009).

Así mismo, nos hemos inspirado en fuentes académicas pertenecientes al campo de los estudios descoloniales para articular las reflexiones que aquí presentamos. Acudimos en particular a los estudios sobre las «epistemologías del Sur»y la descolonización del conocimiento (Santos, 2006).

Y con ese espíritu lúdico que aún es la motivación de algunos investigadores, nos lanzamos a este ejercicio. En la siguiente sección presentaremos los relatos autoetnográficos que describen nuestra experiencia como investigadores en Europa, experiencia en la cual se originan las inquietudes planteadas en este texto. Luego introduciremos brevemente los conceptos clave 
de las epistemologías del sur y la descolonización del conocimiento que han inspirado las reflexiones y conclusiones que se presentan en la sección final.

\section{RELATOS AUTOBIOGRÁFICOS}

a. Mi doctorado en "física de burbujas» (Óscar Enríquez)

\section{El contexto en caricatura}

Nunca había estado en Europa. Europa con mayúscula; la venerable, la de las ciudades monumentales y pueblos pintorescos, los museos fascinantes, la cultura vibrante y el pensamiento Ilustrado; políticamente, la de la democracia social, el Estado de bienestar; la de un capitalismo con rostro más humano que en el otro polo de la hegemonía occidental -Estados Unidos-, la que superó el horror de la guerra construyendo una Unión que es un ejemplo a seguir, la que - a pesar de todo— podría enseñar algo a la izquierda latinoamericana.

Era el final de agosto de 2008. Apenas dos semanas antes había recibido mi título de licenciatura en Ingeniería Mecánica por la Universidad Nacional Autónoma de México (UNAM) cuando partía, becado, a perseguir los títulos de maestría y doctorado en Física de Fluidos. Académicamente, tomaba distancia de la ingeniería para adentrarme en el mundo de la ciencias exactas; si bien la división entre ellas no siempre es definida, en mi mente iba de un mundo donde la pregunta más importante es żcómo?, hacia otro que le da prioridad al żpor qué?

El destino eran los Países Bajos (Holanda para los cuates, aunque no era penal). ${ }^{1}$ Holanda, la de los quesos, tulipanes, zapatos de madera y gente altísima; la progresista, la de la tolerancia, los matrimonios homosexuales, la mariguana cuasilegal, las chicas en las ventanas con luces rojas. Sin embargo,

${ }^{1}$ Para los lectores no mexicanos o no aficionados al futbol, en la copa del mundo de 2014, la selección holandesa eliminó a la mexicana en octavos de final gracias a un penal inexistente, lo cual causó revuelo en redes sociales, con el tema recurrente \#noerapenal. 
me dirigía no a la emblemática y glamorosa Ámsterdam, sino a la última parada del tren hacia el Este: Enschede. Resultó un lugar sosegado y fértil para pensar.

El fin de la infancia

Poco a poco, la caricatura fue deviniendo fotografía nítida. Sí, hay ciudades muy bellas, sin embargo su identidad es empañada por la creciente presencia de cadenas comerciales cuyas inauguraciones suelen ser acompañadas con gran entusiasmo por compradores ávidos de sumarse a las últimas tendencias; los sistemas públicos (como salud y transporte) concesionados a la gestión privada - pues la competencia garantiza mayor eficiencia y mejores servicios-; las universidades se transforman en empresas que venden una marca, donde la retórica de la excelencia, las exigencias de la «economía basada en el conocimiento" y la meritocracia medida en publicaciones y capacidad de atraer dinero determinan las decisiones estratégicas. Da la impresión de que el suelo sobre el que los ciudadanos se sentían firmemente parados es lentamente retirado debajo de sus pies; muchos parecen no darse cuenta por estar tan concentrados en distraerse con las pantallas que los rodean y los que se percatan de que algo extraño está pasando no logran articular una percepción compartida de la situación.

Y entonces, la «crisis de la Eurozona». El contagio desde Estados Unidos. Las noticias no cesan de hablar de los pigs (Portugal, Irlanda, Grecia y España), esos países del Sur, que, según la moral protestante germana y el coro del Norte, vivieron por encima de sus posibilidades reales, gastaron irresponsablemente y ahora deben disciplinarse y ser austeros. La política europea, desde sus partidos e instituciones, muestra impotencia para ofrecer soluciones innovadoras. Se vuelve más que evidente que el sistema financiero, con su ideología y complejidad global, pesa demasiado. Gobiernos técnicos, austeridad, austeridad y más austeridad. Se acentúan las desigualdades. Un destello de esperanza proveniente desde la izquierda «radical» griega es rápidamente sometido y subyugado. Y a pesar de que ese barco hace agua, miles arriesgan y pierden la vida tratando de abordarlo; a los que lo logran no les es-

$178 \frac{\text { SEGUNDO SEMESTRE } 2015}{\text { MIGRACIÓN Y DESARROLLO NÚM. } 25}$ 
pera una bienvenida calurosa. A Europa le cuesta trabajo asumir su responsabilidad en esta otra cara de la crisis.

En las plazas y calles de Madrid, Atenas, Londres, Estambul... las insurrecciones y los disturbios gritan ino! y manifiestan «el hastío por una vida en la que todos estamos solos, solos frente a la necesidad, para cada uno, de ganar su vida, de encontrarse un techo, de alimentarse, de desarrollarse o de cuidarse» (Comité Invisible, 2014: 51). En otros lugares, los que se han dado cuenta de que la verdadera estructura de poder en el mundo actual se encuentra en la infraestructura tecnológica y mercantil (Comité Invisible, 2014: 89), organizan resistencias contra grandes proyectos de infraestructura como trenes de alta velocidad o aeropuertos y contra tratados internacionales de «libre comercio».

Pero la economía debe crecer a como dé lugar. Así que desde 2014 el valor del comercio sexual, de drogas y otros negocios clandestinos debe ser incluido en los cálculos del piB de los países de la Unión Europea (El Financiero, 2014). Los muertos, desplazados y traficados los ponen, principalmente, países que no son de la Unión.

Desde otras geografías, voces que por siglos han resistido los intentos de silenciarlas recobran fuerza una vez más; resuena su palabra con nuevos ímpetus, pues encuentra oídos y corazones que finalmente van sacudiéndose del marasmo inducido por el espejismo de las promesas del progreso, que no pueden más ocultar su falsedad. Voces que asumen el «buen vivir» como afirmación política, que dejan al descubierto la existencia de un sinnúmero de formas de concebir la buena vida, las cuales no necesariamente se oponen entre sí, voces que también advierten sobre la tormenta que viene y que se dan a la urgente tarea de organizar semilleros desde los cuales pueda germinar esa percepción compartida, esencial para que 99 por ciento pueda desembarazarse del dominio del 1 por ciento. Simultáneamente, en Europa hay quien llama a construir la "prosperidad sin crecimiento» (Jackson, 2009) o el «decrecimiento» (D'Alisa, Demaría y Kallis, 2015), quien invita a mirar hacia las «epistemologías del Sur» y a entablar «diálogos de saberes» (Santos, 2006, 2011). 
Y mientras tanto, desde un laboratorio con juguetes de alta tecnología y un ambiente de trabajo inmejorable, un insignificante ingeniero que quiere ser científico estudia el comportamiento de burbujas de gas y se pregunta: ¿̇y tanta (adjetivo) ciencia para qué?

\section{b. iDe dónde es tu doctorado? Experimentando y estudiando el Sur desde el Norte (Victoria Marín-Burgos)}

Mi tensa relación con el viejo continente empezó cuando hice mi maestría en Derecho en la Universidad de Utrecht, Holanda. Corrían los años 20032004, casi un lustro después de terminar mi licenciatura en la Universidad Pontificia Bolivariana en Medellín, Colombia.

Europa siempre deslumbra la primera vez, especialmente si uno llega a Holanda desde la ciudad de Medellín, habiendo vivido en esta última durante los años más cruentos de la violencia que la hicieron mundialmente famosa. La primera impresión es que en Europa, y en particular en los países del norte del continente como Holanda, todo es seguro, todo funciona, las promesas se cumplen y los plazos se respetan. A primera vista no hay una gran diferencia de clases y todos los ciudadanos son «iguales». Personalmente, estaba deslumbrada por el proceso integracionista de Europa, que en aquellos años pasaba por una época dorada. Asimismo, la universidad también me causó gran fascinación en aquel momento. La Universidad de Utrecht me abrió las puertas a formas de aprender y a recursos a los que no había tenido acceso durante mi formación universitaria en Colombia, pues mi experiencia se limitaba a la de víctima del sistema de clase magistral típica de nuestras facultades de derecho en aquel tiempo.

Regresé a Colombia en 2004 con el equipaje cargado de estas románticas memorias europeas, las cuales alimentaron el sueño de volver para hacer mi doctorado. Y así fue, en 2009 me encontraba de nuevo en Holanda empezando un doctorado en Estudios de Desarrollo en la Universidad de Twente, en la pequeña y remota Enschede. Pero aquí la historia cambia de giro. Con los años de interacción con la sociedad y la academia europea el romanticismo

$180 \frac{\text { SEGUNDO SEMESTRE } 2015}{\text { MIGRACIÓN Y DESARROLLO NÚM. } 25}$ 
se acaba. Pasado el enamoramiento, empiezan a hacerse visibles a mis ojos los defectos de esta vieja Europa, a la que le debo la sangre mestiza, y de su academia. Adicionalmente, Colombia, y en particular la Colombia rural, estaban al centro de mi proyecto de doctorado, por lo cual pasé varios meses haciendo trabajo de campo en mi país. La extraña combinación entre la observación cercana de la realidad a través de la experiencia en campo y la reflexión distante sobre dicha realidad una vez de vuelta en Holanda, también contribuyó al cambio de percepción sobre Europa y la ciencia occidental.

Es así como comenzó a aflorar en mí esa tensión interior que vive el mestizo, provocada por la contienda entre su raíz indígena y su raíz europea, y descrita por la socióloga boliviana Silvia Rivera Cusicanqui como la identidad ch'ixi del mestizo (Rivera, 2010). ${ }^{2}$ Empecé a enfrentarme entonces a cuestiones que dieron origen a un diálogo epistemológico con otros investigadores latinoamericanos como Óscar Enríquez, y que están a la base de este esfuerzo por poner en palabras lo que es experimentar el Sur desde el Norte. Cuestiones sobre la hegemonía de la ciencia occidental y la injusticia que ello representa; la falta de elementos de dicha ciencia occidental para observar, estudiar y entender nuestras realidades latinoamericanas y para dialogar con nuestros saberes; el desinterés de la academia europea por otros modos de ver y entender el mundo. De estas cuestiones derivan preguntas como: żsirve el modo de hacer ciencia en el Norte a nuestras realidades latinoamericanas?; ì para qué sirve lo que estoy aprendiendo aquí? , ż por qué y cómo resistirnos a la reproducción hegemónica de la ciencia occidental en nuestros países?

Este desencanto amoroso por Europa y su ciencia me ha enseñado que tomando distancia se puede experimentar y conocer el Sur, desde el Norte. Desde el terreno del colonizador, he sentido y conocido mi Sur como probablemente no lo habría hecho permaneciendo en el terreno del colonizado.

${ }^{2}$ Silvia Rivera Cusicanqui explica que la noción $c h ' i x i$ «obedece a la idea aymara de algo que es y no es a la vez, es decir, a la lógica del tercero incluido». "[L]o ch'ixi conjuga el mundo indio con su opuesto, sin mezclarse nunca con él». Por ello, la socióloga boliviana se refiere a dicha noción como «la traducción más adecuada de la mezcla abigarrada que somos las y los llamados mestizas y mestizos» (Rivera, 2010). 


\section{Epistemologías del SuR y descolonización DEL CONOCIMIENTO}

De acuerdo con Boaventura de Sousa Santos las epistemologías del Sur:

[...] son el reclamo de nuevos procesos de producción, de valorización de conocimientos válidos, científicos y no científicos, y de nuevas relaciones entre diferentes tipos de conocimiento, a partir de las prácticas de las clases y grupos sociales que han sufrido, de manera sistemática, destrucción, opresión y discriminación causadas por el capitalismo, el colonialismo y todas las naturalizaciones de la desigualdad en las que se han desdoblado (Santos, 2011: 35).

A pesar de que existen divergencias entre la teoría de las epistemologías del Sur propuesta por Boaventura de Sousa Santos y los trabajos de reconocidos representantes del pensamiento descolonial latinoamericano como Walter Mignolo o Enrique Dussel (Santos, 2006; Mignolo, 2003; Dussel, 2000), vale resaltar que tanto el pensamiento descolonial latinoamericano como la teoría de las epistemologías del Sur coinciden en la crítica a la ciencia moderna occidental como paradigma hegemónico de conocimiento válido y universal.

Por ello, las reflexiones sobre ciencia y conocimiento en América Latina suscitadas por nuestra experiencia como investigadores de doctorado en una universidad europea que presentamos en la sección final de este ensayo se inspiran en premisas y conceptos de ambas líneas de pensamiento.

En particular, nos han inspirado el concepto de diálogo de saberes, el concepto de lo pluriversal y los argumentos sobre la relación entre capitalismo y colonialismo. Sobre este último argumento, Santos señala que «desde el siglo XV el capitalismo no es pensable sin colonialismo ni el colonialismo es pensable sin el capitalismo» (Santos, 2006). Dicha relación está presente no sólo en los ámbitos cultural, económico y político, sino también en el campo de la ciencia, como lo demuestra Edgardo Lander (2008) en su texto «La ciencia neoliberal».

Santos habla de diálogo de saberes para referirse a la interdependencia entre conocimientos. De acuerdo con Santos, el

$182 \frac{\text { SEGUNDO SEMESTRE } 2015}{\text { MIGRACIÓN Y DESARROLLO NÚM. } 25}$ 
[...] carácter incompleto de todos los conocimientos es la condición para la posibilidad de un diálogo y un debate epistemológico entre ellos. Lo que cada conocimiento aporta a semejante diálogo es la manera en que conduce una cierta práctica para superar una cierta ignorancia. La confrontación y el diálogo entre conocimientos son confrontación y diálogo entre diferentes procesos a través de los cuales las prácticas que son ignorantes de modos diferentes se vuelven prácticas de conocimiento de modos diferentes (Santos, 2011: 36).

Walter Mignolo habla del pensamiento pluriversal para referirse a «formas fronterizas de pensar y de re-inscribir lenguas y cosmologías, saberes y filosofías, subjetividades y lenguas que fueron y continúan siendo demonizadas (esto es, racializadas), desde la posición hegemónica y dominante de la epistemología moderna» (Mignolo, 2008: 36-37).

\section{NUESTRA CIENCIA COLONIZADA Y REFLEXIONES \\ PARA LA RESISTENCIA}

\section{a. Ciencia, tecnología y política}

La investigación científica latinoamericana tiene una "larga» tradición de seguir las pautas marcadas en el Norte. En aras de la pureza, neutralidad y universalismo de las ciencias (especialmente de las consideradas exactas) se asumen sus estándares, se aspira a su reconocimiento y se alinea con los temas que desde allá se decide que vale la pena investigar. Ya en 1969, Óscar Varsavsky denunciaba que

[...] la ciencia actual está adaptada a las necesidades de un sistema social cuyo factor dinámico es la producción industrial masificada, diversificada, de rápida obsolescencia; cuyo principal problema es vender, crear consumidores, ampliar mercados, crear nuevas necesidades —o como quiera decirse- y cuya institución típica es el gran consorcio, modelo de organización y filosofía para las fuerzas armadas, el gobierno, las universidades (Varsavsky, 1969: 22). 
Hoy día este compromiso con tal sistema social no podría ser más explícito en las políticas que orientan el gasto en ciencia e investigación y en las decisiones estratégicas de las universidades. Se puede resumir en el concepto de Sistema Nacional de Innovación, promovido por la Organización para la Cooperación y Desarrollo Económico (OCDE) y adoptado explícitamente por México en su búsqueda por construir una «sociedad de conocimiento». En palabras de uno de los creadores de este concepto: el sistema de innovación «está constituido de elementos y relaciones que interactúan en la producción, difusión y uso de conocimiento nuevo y económicamente útil» (Lundvall, 2002). En su análisis del papel de la institución universitaria en el Norte (Estados Unidos) en la sociedad actual, Bill Readings diagnostica:

[...] la universidad es ahora una corporación burocrática autónoma que responde a la idea de que lo realmente importante en el mundo de hoy es el management económico en vez del conflicto cultural. No le importan más los valores, las ideologías específicas o incluso cosas tan mundanas como aprender a pensar; es simplemente un mercado para la producción, intercambio y consumo de información útil —útil, es decir, para las corporaciones, gobiernos y sus potenciales empleados (Readings, 1997, citado en Harvey, 1998).

Y desde Colombia, un ejemplo de cómo el Sur sigue fielmente al Norte. Un artículo publicado en El Espectador reporta que «de los 189 programas de doctorado que concursaron para recibir becas de Colciencias que permitan financiar a sus estudiantes, sólo 40 pasaron la evaluación preliminar. Ninguno corresponde a ciencias humanas». La explicación del subdirector de Colciencias (Departamento Administrativo de Ciencia, Tecnología e Innovación) es elocuente:

[...] por un lado, argumenta, está la autonomía universitaria para crear los programas que ellas crean que el país necesita. Por otro, argumenta, al gobierno le corresponde priorizar la investigación científica, crear instrumentos para romper asimetrías en los mercados y apoyar programas que son necesarios pero menos atractivos para los estudiantes, como química, matemáticas o física (Correa y Navarrete, 2015). 
Volviendo al Norte, nos encontramos con la forma que puede tomar la integración de las ciencias sociales en equipos «interdisciplinarios» con las exactas dentro del marco actual: asimetría epistémica, presupuestal y de independencia, donde la tajada principal va para las segundas. La ciencia social pareciera ser considerada como un accesorio para que proyectos de exactas (por ejemplo en el campo de nanotecnología) puedan cumplir con el requisito de poder decirse «socialmente responsables y éticos» (Viseu, 2015).

\section{b. ¿Ciencia para la resistencia?}

Para concluir, somos conscientes de que el asunto del que nos ocupamos en este ensayo ha sido estudiado y debatido amplia y profundamente por pensadores del pensamiento descolonial. Por ello, nuestro objetivo es modesto y se limita a compartir las reflexiones que han resultado de nuestras autoetnografías atendiendo al llamamiento hecho por Varsavsky a todos los científicos «para que liberen del culto a una ciencia adaptada a las necesidades de este sistema social y dediquen su talento a preparar científicamente su reemplazo por un sistema nuevo, con una ciencia nueva» (Varsavsky, 1969: 4).

I. Si bien la vigencia del texto de Varsavsky, casi medio siglo después, resulta desoladora, existen notables grupos e iniciativas de científicos que permiten abrigar esperanzas en esa dirección; trabajando con e inspirándose en los viejos maestros de la ciencia de la resistencia: los pueblos indígenas en rebeldía (a los que, como a cualquier maestro, por sabio que sea, tampoco debemos idealizar). Algunos ejemplos son la Unión de Científicos Comprometidos con la Sociedad (UCCS), ${ }^{3}$ la organización Grain, ${ }^{4}$ el grupo ETC ${ }^{5}$ y el proyecto de investigación y activismo llamado EJOLT por sus siglas en inglés

\footnotetext{
3 WWW.uccs.mx

${ }^{4}$ WWW.grain.org

${ }^{5}$ www.etcgroup.org
} 
(Environmental Justice Organisations, Liabilities and Trade), ${ }^{6}$ que realizan diversos trabajos enfocados a replantear la relación de la tecnología con la sociedad, analizar los impactos sociales y ambientales, la producción agroindustrial y la explotación minera y contribuir a la construcción de alternativas. Una de las características importantes de estas iniciativas es su intento por abandonar los paradigmas desarrollistas y construir un marco de equidad epistémica con otros tipos de saberes, sin pretender «ayudar» a que se «desarrollen» y «se saquen a sí mismos de la pobreza». Sin duda habrá una gran cantidad de contradicciones que resolver; los occidentalizados, individualizados y «cientifizados» (Varsavsky, 1969) tenemos mucho que desaprender; además, donde la colonización, el progreso y el desarrollo destruyeron formas de vida, cercenaron y amputaron pasados, hay mucho por re-imaginar y construir.

II. La descolonización de la ciencia y el conocimiento no es materia exclusiva de académicos en el ámbito de las ciencias sociales. Ello es y debe ser objeto de preocupación también entre quienes se ocupan de ciencias exactas, como lo demuestra el hecho de que el diálogo que dio origen a este ensayo se haya dado entre un físico y una investigadora social. De ahí que el diálogo de saberes debe entenderse no sólo como el diálogo entre el saber académico y los otros saberes de nuestros pueblos, sino también como el diálogo entre diferentes campos de la ciencia y diferentes disciplinas. Ello implica entender la interdisciplinariedad no como la subordinación de las ciencias sociales a las ciencias exactas, sino como la interdependencia entre ellas, de manera que pueda repensarse la relación entre ciencias exactas y sociales para que también las primeras puedan estar al servicio de las segundas.

III. Un trabajo interdisciplinario socialmente significativo entre ciencias exactas y sociales requiere de un gran esfuerzo por hacer trabajo de «traducción». Es decir, debe contar con investigadores que posean la capacidad de «hablar», con razonable fluidez, los lenguajes de varias disciplinas a lo largo del espectro exacto-social y la sensibilidad para entender las grandes diferencias en las maneras de abstraer y conceptualizar, es decir, construir mundos

${ }^{6}$ www.ejolt.org. Ejolt es un proyecto de investigación-acción sobre justicia ambiental en el que participan conjuntamente científicos y activistas pertenecientes a organizaciones de diferentes países.

$186 \frac{\text { SEGUNDO SEMESTRE } 2015}{\text { MIGRACIÓN Y DESARROLLO NÚM. } 25}$ 
mirando a través diferentes prismas (véase Santos, 2011, para más detalles sobre traducción entre saberes).

IV. La descolonización de la mente de los investigadores latinoamericanos puede avenirse también en el terreno del colonizador y en algunos casos es posible que sea la distancia del propio origen el factor que dé inicio a este proceso. Adicionalmente, la resistencia contra la hegemonía de la ciencia occidental no necesariamente debe excluir la posibilidad de experimentar y hacer ciencia desde el Norte. De hecho, una lección aprendida de nuestra experiencia es que viviendo la academia del Norte es como hemos empezado a descolonizar nuestra mente. Estando allí es como hemos logrado desmitificar tanto la idea de progreso y desarrollo de Europa, como la superioridad de la ciencia occidental. Estando allí es como hemos logrado develar las falencias de los presupuestos y categorías de la ciencia occidental para entender y enfrentar nuestras realidades, y descubrir el valor de nuestros saberes propios. Quizá no sea casualidad que algunos importantes autores del pensamiento descolonial hayan experimentado la academia del Norte en algún momento o buena parte de su vida académica. ${ }^{7}$ Es más, la presencia de investigadores con vocación a la descolonización de la ciencia en los círculos académicos occidentales facilita la difusión de nuestros saberes, categorías y modos de ver el mundo entre dichos círculos, contribuyendo así al diálogo de saberes y a menguar la hegemonía de la ciencia occidental.

Así mismo, la resistencia contra la hegemonía de la ciencia occidental no necesariamente implica negar o excluir esta última para establecer otro paradigma hegemónico. De hecho, en nuestra experiencia, la academia y las ciencias occidentales nos ofrecieron conocimientos y categorías útiles a nuestras investigaciones y nos permitieron el acceso a oportunidades y

${ }^{7}$ Por ejemplo, Arturo Escobar, desde sus estudios de doctorado hasta el presente, ha estado afiliado a instituciones universitarias de Estados Unidos, manteniendo también una afiliación con instituciones académicas en Colombia. Actualmente es profesor del Departamento de Antropología de la Universidad de Carolina del Norte (UNC), Chapel Hill. Walter Mignolo obtuvo un doctorado en semiótica de la École des Hautes Études de París en 1974 y ha sido profesor en universidades de Estados Unidos desde 1973 (profesor visitante en Indiana y Michigan, carrera académica en Michigan desde 1974 hasta 1992 y desde 1993 es profesor en la universidad de Duke). 
recursos a los cuales tal vez no habríamos tenido acceso en nuestros países. Es por ello que creemos en el diálogo de saberes y consideramos que abogar por un conocimiento y por una ciencia pluriversales implica el reconocimiento de todos los saberes y formas de conocer, incluso la ciencia moderna occidental.

\section{Bibliografía}

Azahua, Marina (2013), Ausencia compartida, México, Fondo Editorial Estado de México.

Butz, David y Kathryn Besio (2009), «Autoethnography», Geography Compass, vol. 3, núm. 5.

Comité Invisible (2014), A nuestros amigos.

Correa, Pablo y Steven Navarrete Cardona (2015), «żEl fin de las humanidades?», El Espectador, en http://www.elespectador.com/noticias/educacion/el-fin-de-humanidades-articulo-591959 (15/10/2015).

D'Alisa, Giacomo, Federico Demaría y Giorgos Kallis (eds.) (2015), Decrecimiento. Vocabulario para una nueva era, Barcelona, Icaria.

Dussel, Enrique (2000) Ética de la liberación en la edad de la globalización y de la exclusión, Madrid, Trotta.

El Financiero (2014), «Sexo y drogas en el PiB de los países de la UE, żpara qué?», en http://www.elfinanciero.com.mx/new-york-times-syndicate/sexo-y-drogasen-el-pib-de-los-paises-de-la-ue-para-que.html (15/10/2015)

Jackson, Tim (2009), Prosperity without Growth: Economics for a Finite Planet, Londres, Earthscan.

Harvey, David (1998) «University, Inc.: The University in Ruins, by Bill Readings», Atlantic-Boston, núm. 282.

LANDer, Edgardo (2008), «La ciencia neoliberal», Tabula Rasa, núm. 9.

LundVAlL, Bengt-Åke et al., (2002), «National Systems of Production, Innovation and Competence Building» Research Policy, vol. 31, núm. 2.

Magritte, René (1928-1929), La Trahison des Images (Ceci n'est pas une pipe), Pintura, Óleo sobre Tela.

Mignolo, Walter D. (2003), Histórias locales / Diseños globales, Madrid, Akal.

$188 \frac{\text { SEGUNDO SEMESTRE } 2015}{\text { MIGRACIÓN Y DESARROLLO NÚM. } 25}$ 
(2008), «Intervenciones descoloniales: Una breve introducción», Tabula Rasa, núm. 9.

Santos, Boaventura de Sousa (2006), Conocer desde el Sur. Para una cultura política emancipatoria, Lima, Fondo Editorial de la Facultad de Ciencias Sociales - UNMSM. (2011), «Epistemologías del Sur», Utopía y Praxis Latinoamericana, vol. 16, núm. 54.

Readings, Bill (1996), The University in Ruins, Boston, Harvard University Press.

Rivera Cusicanoui, Silvia (2010), Ch'ixinakax Utxiwa: una reflexión sobre prácticas y discursos descolonizadores, Buenos Aires, Retazos-Tinta Limón.

VARSAVSKY, Óscar (1969), Ciencia, política y cientificismo, Buenos Aires, Centro Editor de América Latina.

VISEU, Ana (2015), «Integration of Social Science into Research is Crucial», Nature, vol. 525, núm. 7569. 
